\title{
FIRST EIGENVALUE OF THE LAPLACEAN AND TORSION OF PARALLELIZABLE RIEMANNIAN MANIFOLDS
}

\author{
PATRICK GHANAAT
}

(Communicated by Jonathan M. Rosenberg)

\begin{abstract}
Lower and upper bounds for the smallest positive eigenvalue of the Laplacean on a parallelizable Riemannian manifold are combined to obtain an explicit lower bound for the torsion of global orthonormal frame fields in terms of the diameter of the metric.
\end{abstract}

\section{INTRODUCTION AND RESULTS}

Consider a compact, connected $n$-dimensional Riemannian manifold $M$ with metric tensor $g$. We assume that the tangent bundle $T M$ is trivial. Then there exist parallelizations $\omega: T M \rightarrow \mathbf{R}^{n}$ mapping $g$ into the euclidean metric on $\mathbf{R}^{n}$. Such $\omega$ will be called orthonormal. Equivalently, the vector fields $E_{i}$ defined by $\omega\left(E_{i}\right)=e_{i}=i$ th standard basis vector of $\mathbf{R}^{n}$ form an orthonormal frame field on $M$.

The torsion $T$ of $\omega$ is defined by $T(X, Y)=\omega^{-1}(d \omega(X, Y))$ for vector fields $X$ and $Y$ on $M . T$ is the torsion tensor of the flat connection $D$ on $T M$ defined by $D E_{i}=0$. It is well known that a compact Riemannian manifold admits a torsion-free orthonormal parallelization if and only if it is isometric to a flat torus.

In this note we study the relation between isometric invariants of a Riemannian manifold and the torsion of its orthonormal frame fields. Specifically, let $\kappa$ denote the maximum norm of $T$, i.e.,

$$
\kappa=\max \left\{\|T(X, Y)\|: X, Y \in T_{x} M,\|X\|=\|Y\|=1, x \in M\right\} .
$$

The smallest positive eigenvalue of the Laplace operator of $(M, g)$ on functions is denoted by $\lambda_{1}$.

Theorem 1. Let $(M, g)$ be a compact connected Riemannian manifold and $\omega: T M \rightarrow \mathbf{R}^{n}$ an orthonormal parallelization. If for some $p \quad(1 \leq p \leq n-1)$ the Betti number $b_{p}$ of $M$ satisfies $b_{p}>\left(\begin{array}{l}n \\ p\end{array}\right)$, then $\lambda_{1} \leq 100 n^{8} \kappa^{2}$.

Example. Let $M=\Gamma \backslash G$ be a nilmanifold. Then there exist a sequence of Riemannian metrics $\left\{g_{\nu}\right\}$ and a corresponding sequence $\left\{\omega_{\nu}\right\}$ of orthonormal

Received by the editors April 10, 1989.

1980 Mathematics Subject Classification (1985 Revision). Primary 53C20. 
parallelizations on $M$ such that $\kappa^{2} / \lambda_{1}$ converges to zero as $\nu \rightarrow \infty$. For $g_{\nu}$ one can use the almost flat metrics constructed by Gromov and for $\omega_{\nu}$ MaurerCartan forms (see [2]).

Theorem 1 can be combined with lower bounds on $\lambda_{1}$. Here we note the following consequence:

Theorem 2. Under the assumptions of Theorem 1 we have $\kappa d \geq 50^{-n}$, where $d$ is the diameter of $(M, g)$.

The existence of some positive $\varepsilon=\varepsilon(n)$ such that $\kappa d \geq \varepsilon$ also follows from [1].

\section{THE PROOF}

We use tensor notation and the summation convention for the computations. Components of tensors are taken with respect to the orthonormal frame $\left\{E_{i}\right\}$ and coframe $\left\{\omega^{i}\right\}$, where $\omega=\omega^{i} \otimes e_{i}$. For example, $T=T_{i j}^{k} \omega^{i} \otimes \omega^{j} \otimes E_{k}$. Since $g_{i j}=\delta_{i j}$, we need not distinguish between upper and lower indices.

If $\nabla$ denotes the Levi-Cività connection of $g$ then $\nabla-D=S$, where $S$ is given by $S_{i j k}=\frac{1}{2}\left(-T_{i j}^{k}+T_{j k}^{i}-T_{k i}^{j}\right)$. We obtain the following expressions for the exterior derivative $d$ and its formal adjoint $\delta$ acting on a p-form $\alpha$ :

$$
\begin{gathered}
(d \alpha)_{i_{0} \cdots i_{p}}=\sum_{\nu=0}^{p}(-1)^{\nu} \alpha_{i_{0} \cdots i_{\nu} \cdots i_{p}, i_{\nu}}+\sum_{\mu<\nu}(-1)^{\mu+\nu} T_{i_{\mu} i_{\nu}}^{j} \alpha_{j i_{0} \cdots \hat{i}_{\mu} \cdots i_{\nu} \cdots i_{p}} \\
(\delta \alpha)_{i_{2} \cdots i_{p}}=-\alpha_{i i_{2} \cdots i_{p}, i}+T_{i k}^{i} \alpha_{k i_{2} \cdots i_{p}}+\sum_{\nu=2}^{p} S_{i i_{\nu} k} \alpha_{i i_{2} \cdots i_{\nu-1} k i_{\nu+1} \cdots i_{p}} .
\end{gathered}
$$

Here $\alpha_{i_{0} \ldots \hat{i}_{\nu} \ldots i_{p}, i_{\nu}}=E_{i_{\nu}}\left(\alpha_{i_{0} \ldots \hat{i}_{\nu} \ldots i_{p}}\right)=(D \alpha)_{i_{0} \ldots \hat{i}_{\nu} \ldots i_{p} i_{\nu}}$. In particular, Green's Theorem for a vector field $X=X_{i} E_{i}$ can be written

$$
\int_{M} X_{i, i} d V=-\int_{M} T_{k i}^{i} X_{k} d V
$$

with $d V=\omega^{1} \wedge \cdots \wedge \omega^{n}$.

Lemma 1. For vector fields $X$ and $Y$ on $M$,

$$
\begin{aligned}
\int_{M} X_{i, j} Y_{j, i} d V= & \int_{M} X_{i, i} Y_{j, j} d V \\
& +\int_{M}\left(T_{j k}^{k} X_{i, i}-T_{i k}^{k} X_{i, j}+T_{i j}^{k} X_{i, k}\right) Y_{j} d V .
\end{aligned}
$$

Proof. This follows from Green's Theorem and $\left[E_{i}, E_{j}\right]=-T_{i j}^{k} E_{k}$.

Lemma 2. For any harmonic p-form $\alpha$, the $L^{2}$-norm of the covariant derivative D $\alpha$ satisfies $\|D \alpha\|_{2} \leq 10 n^{4} \kappa\|\alpha\|_{2}$. 
Proof. We compute, using the summation convention.

$$
\begin{aligned}
\|d \alpha\|_{2}^{2}= & \sum_{\mu=0}^{p} \int_{M} \alpha_{i_{0} \cdots \hat{i_{\mu}} \cdots i_{p}, i_{\mu}} \alpha_{i_{0} \cdots \hat{i}_{\mu} \cdots i_{p}, i_{\mu}} d V \\
& +\sum_{\mu \neq \nu}(-1)^{\mu+\nu} \int_{M} \alpha_{i_{0} \cdots \hat{i}_{\mu} \cdots i_{p}, i_{\mu}} \alpha_{i_{0} \cdots \hat{i}_{\nu} \cdots i_{p}, i_{\nu}} d V \\
& +2 \sum_{\substack{\rho=0 \\
\mu<\nu}}^{p}(-1)^{\rho+\mu+\nu} \int_{M} \alpha_{i_{0} \cdots \hat{i}_{p} \cdots i_{p}, i_{\rho}} T_{i_{\mu} i_{\nu}}^{j} \alpha_{j i_{0} \cdots \hat{i}_{\mu} \cdots \hat{i}_{\nu} \cdots i_{p}} d V \\
& +\sum_{\substack{\mu<\nu \\
\rho<\sigma}}(-1)^{\mu+\nu+\rho+\sigma} \int_{M} T_{i_{\mu} i_{\nu}}^{j} T_{i_{\rho} i_{\sigma}}^{k} \alpha_{j i_{0} \cdots \hat{\mu}_{\mu} \cdots \hat{i}_{\nu} \cdots i_{p}} \alpha_{k i_{0} \cdots \hat{i}_{p} \cdots \hat{i}_{\sigma} \cdots i_{p}} d V .
\end{aligned}
$$

The first sum is equal to $(p+1)\|D \alpha\|_{2}^{2}$. The absolute values of the third and fourth sums are bounded above by $(p+1) n^{4} \kappa\|\alpha\|_{2}\|D \alpha\|_{2}$ and by $(p+1) n^{5} \kappa^{2}\|\alpha\|_{2}^{2}$, respectively. We apply Lemma 1 and the fact that $\delta \alpha=0$ to obtain an upper bound $6(p+1) n^{4} \kappa\|\alpha\|_{2}\|D \alpha\|_{2}$ for the absolute value of the second sum: By Lemma 1,

$$
\begin{aligned}
\int_{M} \alpha_{i_{0} \cdots \hat{i}_{\mu} \cdots i_{p}, i_{\mu}} \alpha \cdots \hat{i_{\nu}} \cdots i_{p}, i_{\nu} d V= & \int_{M} \alpha_{i_{0} \cdots \hat{i}_{\mu} \cdots i_{p}, i_{\nu}} \alpha{ }_{i_{0} \cdots \hat{i}_{\nu} \cdots i_{p}, i_{\mu}} d V \\
& +\int_{M}\left(T_{i_{\mu} k}^{k} \alpha_{i_{0} \cdots \hat{i}_{\mu} \cdots i_{p}, i_{\nu}}-T_{i_{\nu} k}^{k} \alpha \alpha_{i_{0} \cdots \hat{i}_{\mu} \cdots i_{p}, i_{\mu}}\right. \\
& \left.+T_{i_{\nu} i_{\mu}}^{k} \alpha_{i_{0} \cdots \hat{i}_{\mu} \cdots i_{p}, k}\right) \alpha_{i_{0} \cdots i_{\nu} \cdots i_{p}} d V .
\end{aligned}
$$

Since $\delta \alpha=0$ and $\mu \neq \nu$, we have

$$
\alpha_{i_{0} \cdots \hat{i}_{\mu} \cdots i_{p}, i_{\nu}}= \pm T_{i k}^{i} \alpha_{k i_{0} \cdots \hat{i}_{\mu} \cdots \hat{i}_{\nu} \cdots i_{p}} \pm \sum_{\substack{\rho=0 \\ \rho \neq \mu, \nu}}^{p} S_{i i_{\rho} k} \alpha_{i i_{0} \cdots \hat{i}_{\mu} \cdots \hat{i}_{\nu} \cdots i_{\rho-1} k i_{\rho+1} \cdots i_{p}} .
$$

After substituting these expressions, a straightforward estimate yields the required bound on the second sum. Since $d \alpha=0$, we obtain

$$
\|D \alpha\|_{2}^{2} \leq 7 n^{4} \kappa\|\alpha\|_{2}\|D \alpha\|_{2}+n^{5} \kappa^{2}\|\alpha\|_{2}^{2} .
$$

Lemma 2 follows by completing the square.

Let $K_{\omega} \subset L^{2}\left(\Lambda^{p} T^{*} M\right)$ denote the space of parallel $p$-forms with respect to $D$ and $K_{\omega}^{\perp}$ its orthogonal complement in $L^{2} . K_{\omega}$ is the R-span of $\left\{\omega^{i_{1}} \wedge\right.$ $\left.\cdots \wedge \omega^{i_{p}}: 1 \leq i_{1}<i_{2}<\cdots<i_{p} \leq n\right\}$ and therefore has dimension $\left(\begin{array}{l}n \\ p\end{array}\right)$.

Lemma 3. For any smooth p-form $\alpha \in K_{\omega}^{\perp}, \sqrt{\lambda_{1}}\|\alpha\|_{2} \leq\|D \alpha\|_{2}$.

Proof. Recall that $\lambda_{1}=\inf \left(\|d f\|_{2}^{2} /\|f\|_{2}^{2}\right)$, where the infimum is taken over all functions $f \in C^{\infty}(M)$ such that $\int_{M} f d V=0$. We also note that $\alpha \in K_{\omega}^{\perp}$ 
if and only if each component function of $\alpha$ is orthogonal to the constants in $L^{2}$. Thus,

$$
\|\alpha\|_{2}^{2}=\sum_{i_{1}, \ldots, i_{p}}\left\|\alpha_{i_{1} \cdots i_{p}}\right\|_{2}^{2} \leq \lambda_{1}^{-1} \sum_{i_{1}, \ldots, i_{p}, j}\left\|\alpha_{i_{1} \ldots i_{p}, j}\right\|_{2}^{2}=\lambda_{1}^{-1}\|D \alpha\|_{2}^{2} .
$$

The hypothesis $b_{p}>\left(\begin{array}{l}n \\ p\end{array}\right)$ implies that there exists a nonzero harmonic form in $K_{\omega}^{\perp}$. Lemmas 2 and 3 now imply Theorem 1 . To prove Theorem 2 we recall [1, Theorem 3.1]: If $\kappa d \leq 0.05$ then $\lambda_{1} \geq 20^{-n} d^{-2} \lambda_{1}^{*}$, where $\lambda_{1}^{*}$ is the smallest positive eigenvalue of the Laplacean on the $n$-dimensional euclidean unit ball under Neumann boundary conditions. By a result of Payne and Weinberger [3], $\lambda_{1}^{*} \geq \pi^{2} / 4$, and Theorem 2 follows from Theorem 1 .

\section{REFERENCES}

1. P. Ghanaat, Almost Lie groups of type $\mathbf{R}^{n}$, J. Reine Angew. Math. (to appear).

2. M. Gromov, Almost flat manifolds, J. Differential Geom. 13 (1978), 231-241.

3. L. E. Payne, H. Weinberger, The optimal Poincaré inequality for convex domains, Arch. Rat. Mech. Anal. 5 (1960), 282-292.

4. E. Ruh, Almost Lie groups, Proc. International Congress of Math., Berkeley (1986).

Mathematisches Institut Der Universität, Rheinsprung 21, CH-4051 Basel, SWITZERLAND 